\title{
La calidad del equipamiento turístico
}

\author{
María de los Ángeles González Cobrero \\ Escuela Universitaria de Turismo \\ Universidad de Sevilla \\ Responsable de calidad del Hotel Bécquer
}

(Sevilla)

\begin{abstract}
Resumen
Si cuando llega a la ciudad, no encuentra un medio de transporte al hotel. Si le roban la cartera en la estación de tren, si no encuentra nadie que hable su idioma y que le ayude a solucionar su situación, si llega al hotel y es atendido con mala cara por el recepcionista...En fin, han de conjugarse toda una serie de elementos para conseguir la satisfacción total del cliente. Por decirlo de otro modo, hay calidad en el servicio y de forma sostenible en el tiempo (calidad total), si todos los agentes implicados -clientes, empresa prestadora, entorno social y medioambiental-, aportan y reciben en el círculo de la prestación.
\end{abstract}

\section{Palabras claves}

Turismo / Calidad / Sistemas de calidad

\section{Introducción}

Tras varios años de crecimiento cuantitativo, la industria turística, se encuentra en una encrucijada, donde las cuestiones de rentabilidad privada han pasado a un segundo plano, exigiendo, el logro de competitividad en productos y regiones turísticas en un contexto de creciente preocupación por los impactos económicos y medioambientales del turismo.

En la década de los 60, el boom turístico mundial, motivó una generación masiva de productos turísticos ofreciendo al cliente, por tanto, unos productos totalmente estandarizados y por consiguiente, muy rígidos.
Si embargo, en el contexto actual, el producto debe ser cada vez más especializado y adaptado a unas necesidades de demanda cada vez más complejas y diversas que nos exige un conocimiento profundo del mercado y una mayor flexibilidad en la adaptación a las exigencias de la demanda en cuanto a la organización, producción y distribución del producto turístico.

En este entorno cada vez más competitivo, en el que el factor precio ya no es el único determinante para la elección del destino, la calidad supone la apuesta decididamente más lógica para hacer frente a los retos más importantes a los que se enfrentan las empresas turísticas.

El grado de desarrollo y la transformación que a escala mundial está experimentando la industria turística, está afectando al usuario del producto que cada vez más "profesionalizado", exige prestaciones de servicio con un mayor grado de calidad y una mayor adecuación de la oferta al nivel de la demanda suponiendo para el empresario español la exigencia de una gestión empresarial que conjugue de la manera más perfecta posible los parámetros calidad, competitividad, rentabilidad y sostenibilidad. Es decir, exige la adopción de métodos y procedimientos que garanticen un óptimo equilibrio entre los cuatro parámetros conjugados.

Resumiendo, hoy en día es primordial conseguir ofrecer un servicio turístico de calidad sostenible en el tiempo, evaluado por la percepción del cliente y en el que todos los elementos implicados sean tanto receptores como contribuyentes a la prestación.

Y esto es aplicable a cualquier tipo de turismo: turismo de sol y playa, deportivo, cultural... pero quizás con más hincapié cuando hablamos de ciudades históricas, ya que nos referimos a un turista generalmente exigente y acostumbrado viajero que nos va a demandar, como algo a lo que está acostumbrado, unos servicios de calidad.

El crecimiento del turismo va a ir paralelo con la calidad del equipamiento turístico de la ciudad, y no 


\begin{abstract}
Hay que implicar y concienciar a las personas -profesionales del sector o no- de su importancia en la prestación de un turismo de calidad y hacerles ver las ventajas que ese turismo bien llevado y planificado conlleva. Podemos tener una ciudad preciosa, y con una oferta cultural muy interesante, pero si no disponemos de las infraestructuras adecuadas difíilmente atraeremos turismo dispuesto a quedarse el mayor tiempo posible y a volver
\end{abstract}

debemos olvidar que el turismo cultural, es un producto actualmente "de moda", no sólo para el viajero, sino para los proveedores, que ven en este crecimiento del turismo de ciudad, una nueva y suculenta fuente de ingresos. Eso por otra parte implica competencia y saber hacer. La mejor oferta en todos los sentidos ( relación calidad-precio, infraestructura general,...) será la que "se lleve el gato al agua".

Por último, hacer mención de que cuando hablamos de calidad del equipamiento turístico, hemos de incluir en este equipamiento turístico, tanto la infraestructura puramente turística, como la que no lo es, siempre y cuando esté en contacto con el cliente final.

\section{Nociones generales de calidad y calidad total en el ámbito turístico}

Podemos definir el término calidad de varias formas, pero para mí, el más acertado es aquel que nos habla de la prestación objetiva de un servicio, evaluado a través de cuestionarios que nos permitan conocer la satisfacción del cliente.

Esta medición, es en ciertos casos muy compleja, entre otras cosas, por que el producto turístico conlleva una serie de cualidades que dificultan esta medición. Es intangible, subjetivo, instantáneo y heterogéneo.

Es intangible por su dificultad en ser mostrado antes de su uso, es subjetivo, porque va a estar totalmente motivado por el estado de ánimo del cliente, es heterogéneo porque está compuesto de muy diversos factores y servicios y es instantáneo, porque se produce y consume en el acto. Para que lo entiendan un poco mejor, cuando un agente de viajes nos vende un paquete turístico a Canarias, nos está vendiendo un avión, un hotel y una serie de servicios adicionales que no podemos conocer más que por fotografías y una vez se está prestando el servicio, de ahí se produce su instantaneidad. Si no nos gusta, difícilmente podremos devolverlo. Por otra parte, esos servicios turísticos no son homogéneos en cuanto a su contenido, están formados por la combinación de una serie de elementos tangibles (la habitación del hotel, el asiento del avión...) y otros intangibles (componente humano y claro determinante en muchos casos de la percepción de calidad que el cliente tiene el servicio), además de por servicios turísticos y no turísticos.

No basta con que tengamos una infraestructura turística de primera (hoteles de lujo, buenos aeropuertos...) sino que también hemos de contar con la infraestructura no turística y/o de servicios en general (hospitales, bancos...), con la preparación para recibir al turista, tanto de las personas claramente implicadas (trabajadores turísticos propiamente dichos) como del resto del entorno social en general y como no, con un servicio más a prestar al turista: la seguridad ciudadana. Hay que implicar y concienciar a cada parte humana (participe o no en la prestación del servicio propiamente turístico) de su importancia en la prestación de un turismo de calidad y hacerles ver las ventajas que ese turismo bien llevado y planificado conlleva. Podemos tener una ciudad preciosa, y con una oferta cultural interesantísima, pero si no disponemos de los servicios adecuados difícilmente atraeremos turismo, o quizás, si tenemos algo "único" sí lo atraigamos, pero el turista que venga, vendrá "obligado" por su interés en conocer algo, se quedará el menos tiempo posible y seguramente no volverá.

Si cuando llega a la ciudad, no encuentra un medio de transporte al hotel. Si le roban la cartera en la estación de tren, si no encuentra nadie que hable su idioma y que le ayude a solucionar su situación, si llega al hotel y es atendido con mala cara por el recepcionista... En fin, han de conjugarse toda una serie de elementos para conseguir la satisfacción total del cliente.

Por decirlo de otro modo, hay calidad en el servicio y de forma sostenible en el tiempo (calidad total), si todos los agentes implicados -clientes, empresa prestadora, entorno social y medioambiental-, aportan y reciben en el círculo de la prestación. 
Veamos con algunos ejemplos algunas situaciones negativas con las que nuestro turista se encuentra de vez en cuando:

Calidad del servicio turístico

1 er Fracaso

Nuestro cliente, acude a una agencia de viajes, para solicitar la reserva de una habitación doble en la ciudad de "X"...El agente de viajes que les atiende está muy ocupado y ni siquiera les ofrece asiento.

$2^{\circ}$ Fracaso

Una vez llega a la ciudad, coge un taxi para trasladarse al hotel y el taxista le hace "un recorrido turístico de 1/2 hora"(con el consecuente aumento de precio) cuando se había informado que no se tardaba más de 10 minutos...

3er Fracaso

Por fin, en el hotel...entrega el bono en recepción y el recepcionista "amablemente" les indica que debido a una situación de "overbooking" han de trasladarse a otro hotel, de similar categoría, pero en las afueras de ciudad..

$4^{\circ}$ Fracaso

Después de mucho discutir... acepta el cambio, y se va al nuevo hotel. La atención que recibe en el nuevo es buena, pero cuando llega a la habitación (predispuesto a encontrar fallos), descubre que el cuarto de baño es muy pequeño y con manchas de humedad...

¿Cómo creen que se sentirá nuestro cliente?

- ¿Influirá lo ocurrido en su imagen de la ciudad visitada?

- ¿Recomendará la visita de la misma...?

\section{Objetivos de la calidad}

Retomando la frase anterior... : Todos los agentes implicados han de dar y recibir...ंQué quiere decir esto?. Pues que "tener calidad" no sólo significa implantar un sistema determinado de gestión, utilizar una determinada herramienta o realizar unos documentos, es mucho más que eso, es conseguir unos objetivos complejos que son:

- Satisfacción del cliente por el servicio o producto recibido medida en función de sus expectativas (subjetividad).

Hemos de preguntarnos: i Que espera recibir el turista del producto que se le ofrece? Sus expectativas serán lógicamente distintas según él mismo. No esperará lo mismo un turista que venga a España a pasar una semana en la Costa del Sol que el que venga a realizar un circuito por Córdoba, Granada y Sevilla, aunque pueden coincidir en ciertas cosas, habrá otras en las que difieran.

Centrémonos, por ejemplo, en las ciudades históricas. ¿Qué creéis que espera el turista que viene a una ciudad como Baeza?

Si cumplimos esa expectativas en un 70/80\% (dejemos un margen de error humano del 10/20\%) tendremos a un turista muy satisfecho, si las cumplimos en un 50/60\% la satisfacción no será plena, pero estaremos en el límite, debemos mejorar en el futuro. Todo lo que baje de esos porcentajes supondrá una mala gestión de la calidad turística.
Es decir, hemos de conocer las expectativas del cliente y traducirlas en productos/servicios que le satisfagan totalmente, completándose el ciclo con la medición del nivel de satisfacción, y la puesta en marcha de mecanismos de mejora que permita aumentar ese nivel.

- Fidelización del cliente. Es la consecuencia directa de conseguir cumplir las expectativas del cliente. Un cliente satisfecho, volverá a visitarnos y lo que es más importante, será una fuente de publicidad y difusión que nos traerá nuevos visitantes.

- Satisfacción del personal prestador del servicio o personal de contacto. Hay calidad cuando los empleados y/o prestadores están motivados y cuentan con los medios para prestar el servicio adecuadamente, cuando se sienten parte de la empresa y sienten que sus opiniones son tenidas en cuenta por el empresario.

Hemos de pensar, que este personal, es en la mayoría de los casos, el más cercano al cliente y el que mejor que nadie conoce sus deseos y su opinión de los servicios, es la "imagen" de la empresa. El empresario ha de tener en cuenta su opinión, siendo esta quizás una de las mejores formas de motivación.

- Satisfacción de la empresa prestadora de los servicios, y por tanto, de sus accionistas.

En este punto, es necesario considerar, que el empresario estará dispuesto a realizar mejoras de calidad en su empresa, si existe una adecuación entre los costes y la rentabilidad que se va a recibir a cambio, y en la mayoría de los casos, aunque erróneamente y sólo en principio se acepta la implantación de un sistema de calidad, pensando en los beneficios económicos que una certificación de calidad puede traer.

Por ejemplo, en una provincia andaluza, se está implantando actualmente en varios hoteles el sistema de calidad del ICHE. En un principio, fueron bastantes los hoteles que se apuntaron a esa iniciativa, pero con el tiempo, el número se ha reducido. ¿Porqué? Pues en muchos casos, cuando decidieron apuntarse no se dieron cuenta, o no les fue explicado, las dificultades que la implantación conlleva, que no se trata de algo puntual, sino de algo a mantener en el tiempo, no vieron las dificultades de cambiar un sistema de trabajo en muchos casos mantenido durante años. Pensaban sólo, en la obtención de una marca que les ayudaría como arma de marketing y venta, y aunque realmente es uno de los beneficios de la implantación, conlleva una serie de dificultades que no evaluaron en su momento o que cómo ya he dicho no les fue debidamente explicado.

- Por otra parte, en muchos casos y a la vista de la dificultad de la implantación, se optó por abandonar justificando la decisión en la falta de tiempo, en pensar que si hasta ahora nos ha ido bien con el sistema que tenemos que necesidad hay de cambiarlo, y para muchos la mejor manera de comprobarlo es según la ocupación del hotel, que necesidad tenemos de cambiar el sistema. ¿Qué mejor garantía de calidad que 
una ocupación alta? (y en cierto modo es así, si el cliente no está satisfecho, el hotel no se llena).

- En fin, es necesario también educar al empresario y hacerle ver que la puesta en marcha de un plan de mejora global de gestión de la empresa (calidad total), ha de contar, con el compromiso de la dirección y demás mandos y con una activa implicación de todos los que trabajan en al empresa, de modo que hacer bien las cosas desde el principio y a la primera, y orientarse hacia la satisfacción del cliente no sea algo excepcional sino que forme parte del trabajo cotidiano y esté arraigado como cultura de empresa

- Sostenibilidad medioambiental y entorno social. El turismo cultural ha conocido un importante incremento en la última década, pero para evitar sus efectos negativos es necesario asumir que la capacidad de la carga turística de las ciudades históricas es limitada, ya que los recursos de patrimonio cultural son frágiles y no renovables. Sostenibilidad significa, lograr modelos turísticos integrados en la economía y sociedad local, respetuosos con el patrimonio urbanístico y el medio ambiente, así como preocupados por las nuevas demandas de accesibilidad y movilidad.

En definitiva, integrar el turismo en la ciudad y regular los flujos de visitantes, eso es gestionar calidad.

Hemos de considerar, que aunque el turismo constituye un pilar importante en la economía de muchas ciudades históricas, sino se consigue una integración adecuada del mismo se pueden convertir en un continúo foco de conflictos y producir tanto efectos positivos como negativos, como algunos de los que podemos ver en el cuadro siguiente:

Efectos positivos

Dinamización de la actividad económica de la ciudad.

Generador de riqueza y empleo. Incrementa la renta de la población local y genera aumento de los ingresos de las corporaciones locales.

Potencia el desarrollo de los sectores de actividad turísticos y no turísticos. Creando puestos de trabajo directos e indirectos.

Introduce nuevos hábitos y formas de vida entre la población autóctona.

Contribuye a reforzar el mantenimiento de los monumentos, ayuda a revalorizar recursos locales que estaban infrautilizados y propicia la aparición de una oferta cultural diversificada.

\section{Efectos negativos}

Si la ciudad depende demasiado el turismo, un descenso de la afluencia de turista puede provocar una crisis.

Por otra parte, encarece el coste de la vida para los "locales", aumenta la inflación y hace aparecer la especulación.

Pueden desaparecer los viejos comercios y por lo tanto, los empleos que los mismos generaban.

Incrementa el tráfico, los lugares de ocio y produce alboroto, que puede molestar a la población residente y provocar su abandono de las zonas históricas hacía otras menos turísticas.
Hablamos, pues de calidad total cuando la calidad se entiende como un sistema a mantener en el tiempo, no como la consecución de un certificado en un momento determinado. Por supuesto, este mantenimiento es difícil hablando de turismo, porque ya sabemos de la dificultad de conseguir el "defecto cero" cuando de percepción y prestación humana se habla. Sin embargo, lo que sí prevén los sistemas de calidad son los mecanismos de recuperación y de corrección de errores, con una continua autoevaluación en la prestación del servicio. Si existe un error, si el cliente tiene razón en no sentirse plenamente satisfecho, la deficiencia debe corregirse enseguida.

\section{Sistemas de calidad}

¿Porqué se implanta un sistema de calidad? ¿Qué sentido tiene para la iniciativa privada complicarse en la implantación de un sistema nuevo de gestión y trabajo si como decíamos antes, la ocupación es alta y estamos en años de bonanza económica y de crecimiento del turismo?

Pues yo creo que es una forma de luchar con la competencia venidera, decía al principio que los nuevos mercados vienen pujando fuerte y ofrecen servicios excelentes y de reciente creación. La única manera en muchos casos de luchar contra esta competencia es con una oferta global y estandarizada que garantice al cliente unos servicios excelentes. $Y$ eso es desde mi punto de vista, lo que pretenden conseguir los sistemas de calidad, la estandarización de los servicios desde cada uno de los sectores integrantes del turismo, la garantía al cliente de que la oferta que se va a encontrar, cuenta con una serie de servicios mínimos iguales a todos los establecimientos.

Por otra parte, no es necesario que el gestor se incline por uno de los planes de calidad existentes, pudiendo poner en práctica el suyo propio. La única desventaja que esto representaría sería que este no estaría reconocido oficialmente, perdiendo así la posibilidad de utilizarlo como arma de marketing y ventas.

Actualmente, se están aplicando en España, principalmente, dos sistemas de calidad distintos las normas ISO9000 y el PCTE, sistema creado por el ICHE.

Ambas normas suponen para la empresa, la obtención de un certificado de calidad, una vez implantado el sistema según las normas especificadas por cada uno de ellos y pasada una auditoría externa e imprevista. Generalmente este certificado es válido por un año renovable, previa nueva auditoría.

Ambas, especifican los requisitos que debe cumplir un sistema de calidad para asegurar que los servicios entregados por el proceso en cuestión están en línea con los requisitos específicos y acordados con el cliente. Constituyen pues un mecanismo razonable por el cual la dirección de una empresa puede de 
forma sistemática garantizar que los servicios que se prestan a los clientes están en línea con sus requerimientos, además de aplicar la idea de la mejora continua a todas las actividades vinculadas al sistema.

¿Cuál es la diferencia entre uno y otro y qué ha motivado la aparición del PCTE, si ya existía un sistema de calidad reconocido y eficaz?

Pues bien, las normas ISO 9000 nacieron originariamente aplicadas a la industria a partir de las normas BS 5750, por lo que su utilización en el sector servicios es muy reciente. La falta inicial de adaptación al sector de servicios unido al lenguaje más orientado a la Industria, hicieron que en el caso del sector turístico español sea muy escasa la aplicación de esta normativa y facilitaron la aparición del sistema creado por el ICTE, específico y común para todos los sectores turísticos.

Sin embargo, a partir de Enero de 200 I han entrado en vigor la nueva familia de normas de calidad ISO9000, revisadas con el fin, entre otras cosas, de conseguir una mayor interpretabilidad u orientación hacia organizaciones de prestación de servicios y de no quedarse al margen de la evolución de los conceptos y enfoques de gestión, en sintonía con los llamados "modelos de excelencia", que muestras, entre otros aspectos, un compromiso más sólido en la búsqueda de la satisfacción de los clientes, dentro de una dinámica de mejora continua de la organización. En definitiva, ya no se trata tanto de asegurar los procesos que discurran por el cauce correcto, como de permitir su mejora continua.

\section{¿En qué consiste un plan de calidad?}

Hasta ahora hemos visto que como aspectos más positivos de los sistemas de calidad, podemos citar, la creación de una percepción de seguridad para el consumidor, que puede confiar en que las empresas, productos o servicios garantizados, van a cumplir los requerimientos establecidos, evitando así, sorpresas, insatisfacciones de expectativas o problemas imprevistos y de cara al proveedor, estos sistemas de calidad suponen un importante herramienta de marketing a usar de cara a captar clientes y a mantener a los antiguos ante la competencia.

También hemos visto que son una herramienta útil para motivar y mejorar la comunicación interdepartamental, además de adecuar y motivar a los trabajadores dentro de sus puestos de trabajo.

Por último, y como una de las ventajas más importantes, contar con una herramienta que permita la mejora continúa de la organización en busca de la calidad total.

Por otra parte, como aspectos negativos hemos señalado que son sistemas difíciles de aplicar, pues en muchos casos significan un cambio importante en la forma de trabajar, que pueden derivar en una exce- siva burocratización del trabajo y que en algunos casos conllevan gastos iniciales y rechazo tanto por parte del empresario como de los empleados.

Pero aún no hemos visto, que es lo que se hace para implantar un plan de calidad.

Normalmente se comienza por una primera auditoría interna que llevada según las normas que el plan de calidad nos indica, nos permite conocer a que distancia estamos de conseguir la certificación de calidad.

Lógicamente esta auditoría ha de hacerse con todo el personal o bien con equipos de calidad seleccionados del mismo. No hemos de olvidar que se pretende la integración y el interés de todos en la implantación y desarrollo del plan. Generalmente al principio, suele haber reacciones adversas motivadas por la dificultad que a primera vista representa, pero con el tiempo y al hacerles ver las ventajas que para ellos mismos la implantación conlleva suelen sentirse mucho más involucrados.

Los resultados de la auditoría han de estudiarse de manera global y por servicios, de modo que según la valoración obtenida, se puedan extraer conclusiones sobre las situación de la Calidad del servicio y las áreas de mejora que es necesario desarrollar para mejorar la calidad.

El siguiente paso dentro del ciclo de gestión de la calidad, sería la implantación y utilización de las herramientas de gestión de la calidad, que son:

\section{Planificación de la mejora.}

Se entiende como área de mejora aquel proceso o servicio que no cumple con los estándares de calidad requeridos y es susceptible de mejora. Sobre esta área estableceremos un plan de mejora que nos ayude a conseguir los mencionados estándares y un sistema de indicadores que nos permita comprobar la implantación del plan de mejora y su eficacia.

\section{Sistema de indicadores.}

Son la herramienta de medición que permite hacer el seguimiento de la calidad que se está ofreciendo al cliente, así como las desviaciones temporales que se pueden producir en el servicio sometido a estudio. De esta forma, los indicadores permitirán tomar medidas preventivas y/o correctoras para asegurar la mejora en el tiempo.

Tipos de indicadores:

- Indicadores de calidad del proceso. Reflejan el nivel de cumplimiento de las especificaciones previstas en la realización de las actividades empresariales. Se basa en datos generados en la realización de dichas actividades. (P. ej: tiempo en que se tarda en hacer el chek-in)

- Indicadores de calidad del producto o servicio. Reflejan las características del producto/servicio final entregado al cliente. Se basa en datos de inspec- 
ción • verificación recogidos internamente. (P. ej: $N^{\circ}$ de clientes que han tenido que esperar más de diez minutos para realizar el chek-in)

- Indicadores de percepción del cliente. Reflejan la opinión del cliente respecto al producto o servicio recibido. Se pueden recoger a través de encuestas. (P. ej: $N^{\circ}$ de quejas de clientes por tiempos de espera).

Un sistema de indicadores de calidad completo debe contemplar los tres tipos de indicadores, de manera que exista un control global de las actividades empresariales y de los resultados del servicio frente al cliente.

\section{Sistema de gestión de quejas y sugerencias.}

Mecanismo sistematizado que permite recoger $y$ tener en consideración la opinión de los clientes, tanto internos como externos, con la finalidad de mejorar la gestión del Sistema de calidad del establecimiento.

Objetivos:

- Recoger del cliente toda la información relevante.

- Mejorar la atención y servicio al cliente.

- Personalizar el servicio.

- Recuperar la satisfacción de los clientes que hayan percibido los problemas.

- Capacitar a los trabajadores para la resolución de los problemas

\section{Sistema de encuestación.}

Sistema que permite a través de un cuestionario obtener información sobre la satisfacción del cliente de forma práctica, eficaz y eficiente.

Objetivos

- Conocer la opinión del cliente sobre los diversos servicios que ofrece el establecimiento.

- Impulsar la mejora continua de los servicios ofrecidos al cliente, guiada por la opinión ofrecida.

- Hacer un seguimiento sistemático del nivel de satisfacción alcanzado por la clientela.

Estos dos últimos sistemas, no son otra cosa que el estudio de las expectativas y nivel de satisfacción del cliente del que ya hemos hablado.
Una vez este proceso está en marcha podemos decir que estamos empezando a gestionar calidad, obtenemos unos resultados, los estudiamos y si es necesario establecemos los planes necesarios para mejorarlos. Pero aún queda un paso, que quizás sea el más arduo y al que todos somos más reacios: la Gestión Documental

Esta gestión no es otra cosa que una metodología general establecida por el plan de calidad para la elaboración, documentación y gestión de procedimientos y registros de una forma sistemática y concreta y aplicada a todas las actividades del establecimiento que tenga incidencia en el sistema de calidad. En fin nos "obliga" a que tengamos un mínimo de procesos y registros documentados y formateados según la norma.

Con esto tendríamos el sistema de calidad en marcha. Una vez que la empresa considera que ya está preparada para recibir la certificación de calidad, el siguiente paso es solicitar la auditoría externa que facilitará el informe sobre al situación del establecimiento y la autorización para la concesión del certificado de calidad.

El uso de la marca de calidad obliga al establecimiento a la gestión de calidad y a someterse a nuevas auditorías anuales y de renovación, tanto con aviso previo cómo de carácter imprevisto.

Algunas conclusiones:

- Hay calidad en el servicio y de forma sostenible en el tiempo (calidad total) siempre y cuando todos los agentes implicados aporten y reciban en el círculo de la prestación.

- Definitivamente la calidad del servicio no sólo depende del equipamiento puramente turístico.

- Las actuaciones para mejorar la calidad han de provenir tanto desde la iniciativa privada como de la pública

- Toda la población ha de ser consciente de la importancia de "su destino turístico".

\section{Bibliografía}

MARCHENA GÓMEZ, MANUEL (ed.) Turismo Urbano y patrimonio cultural -Una perspectiva Europea. Sevilla: Patronato de Turismo de Sevilla

Estudios Turísticos. Nº 123 y I39. Es una revista editada por el Instituto de Estudios Turísticos. Sin un periodicidad muy concreta

BIGNÉ, J. ENRIQUE; LÓPEZ, DIEGO. Planificación y comercialización turística. Universitat Jaume-I
La calidad, - Un reto para el turismo- .Editado por la OMT sobre un seminario sobre calidad [fotocopia de las ponencias]

Plan de calidad Turística Española. Publicado por el Instituto de la Calidad Turística Española. ICTE 